\title{
Nonlinear long-wave deformation and runup in a basin of varying depth
}

\author{
I. Didenkulova \\ Institute of Cybernetics, Tallinn University of Technology, Akadeemia tee 21, 12618 Tallinn, Estonia \\ Institute of Applied Physics, Uljanov Street 46, 603950 Nizhny Novgorod, Russia
}

Received: 6 October 2008 - Revised: 17 December 2008 - Accepted: 17 December 2008 - Published: 29 January 2009

\begin{abstract}
Nonlinear transformation and runup of long waves of finite amplitude in a basin of variable depth is analyzed in the framework of 1-D nonlinear shallow-water theory. The basin depth is slowly varied far offshore and joins a plane beach near the shore. A small-amplitude linear sinusoidal incident wave is assumed. The wave dynamics far offshore can be described with the use of asymptotic methods based on two parameters: bottom slope and wave amplitude. An analytical solution allows the calculation of increasing wave height, steepness and spectral amplitudes during wave propagation from the initial wave characteristics and bottom profile. Three special types of bottom profile (beach of constant slope, and convex and concave beach profiles) are considered in detail within this approach. The wave runup on a plane beach is described in the framework of the CarrierGreenspan approach with initial data, which come from wave deformation in a basin of slowly varying depth. The dependence of the maximum runup height and the condition of a wave breaking are analyzed in relation to wave parameters in deep water.
\end{abstract}

\section{Introduction}

Giant sea waves (storm waves, tsunamis) approaching the coast often lead to the destruction of coastal infrastructure and the loss of life. These waves have various origins: strong storms and cyclones, underwater earthquakes, and sub-aerial and sub-marine landslides. Recent such events are the catastrophic tsunami in the Indian Ocean on 26 December 2004 (Lay et al., 2005; Glimsdal et al., 2006), hurricane Katrina (28 August 2005) in the Atlantic Ocean (Kim et al., 2008), and typhoon "Nargis" which occurred on 3 May

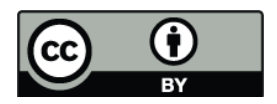

Correspondence to: I. Didenkulova (ira@cs.ioc.ee)
2008 in the Indian Ocean. Volcanic eruptions, such as the 1883 Krakatau event (Simkin and Fiske, 1983; Choi et al., 2003; Pelinovsky et al., 2005), or the collapse of the Cumbre Vieja volcano at La Palma, Canary Islands (Ward and Day, 2001; Mader, 2001; Pararas-Carayannis, 2002), huge landslides such as the Storegga slide which occurred in Norway 8000 BP (Bugge et al., 1988), and asteroids entering the sea (Hills and Gods, 1998; Ward and Asphaug, 2000; Kharif and Pelinovsky, 2005) can also generate huge waves which are recorded world-wide. Unusual and short-lived freak or rogue waves occur on the coast and result in the loss of lives every year (Kharif and Pelinovsky, 2003; Didenkulova et al., 2006a; Kharif et al., 2009). High-speed ferries operating in the coastal zone induce large waves, which can lead to a beach erosion and seabed change (Soomere, 2007; Parnell et al., 2008).

The prediction of the possible flooding of the adjacent land and the properties of the water flow at the coast is an important practical task for coastal engineering. That is why there are numerous empirical formulas describing runup characteristics in the engineering literature (see, for example, Le Mehaute et al., 1968; Stockdon et al., 2006). It is difficult to obtain solutions of the basic 3-D hydrodynamic equations with arbitrary nonlinearity and dispersion. Thus, nonlinear shallow water theory based on hydrodynamic Euler equations for long-wave approach in an ideal fluid is an appropriate model for examining long wave propagation and runup on a beach. The runup problem within this theory has a rigorous solution only for the case of a constant slope beach profile (Carrier and Greenspan, 1958). An important outcome of this solution is that linear theory can be used to describe extreme characteristics of the wave runup on a beach (Synolakis, 1991; Didenkulova et al., 2007).

Real bottom bathymetry is much more complicated than a plane beach, and rigorous solutions of runup stage should be matched with characteristics of an incident wave, which pass long distances from the open ocean to the beach. In the

Published by Copernicus Publications on behalf of the European Geosciences Union and the American Geophysical Union. 

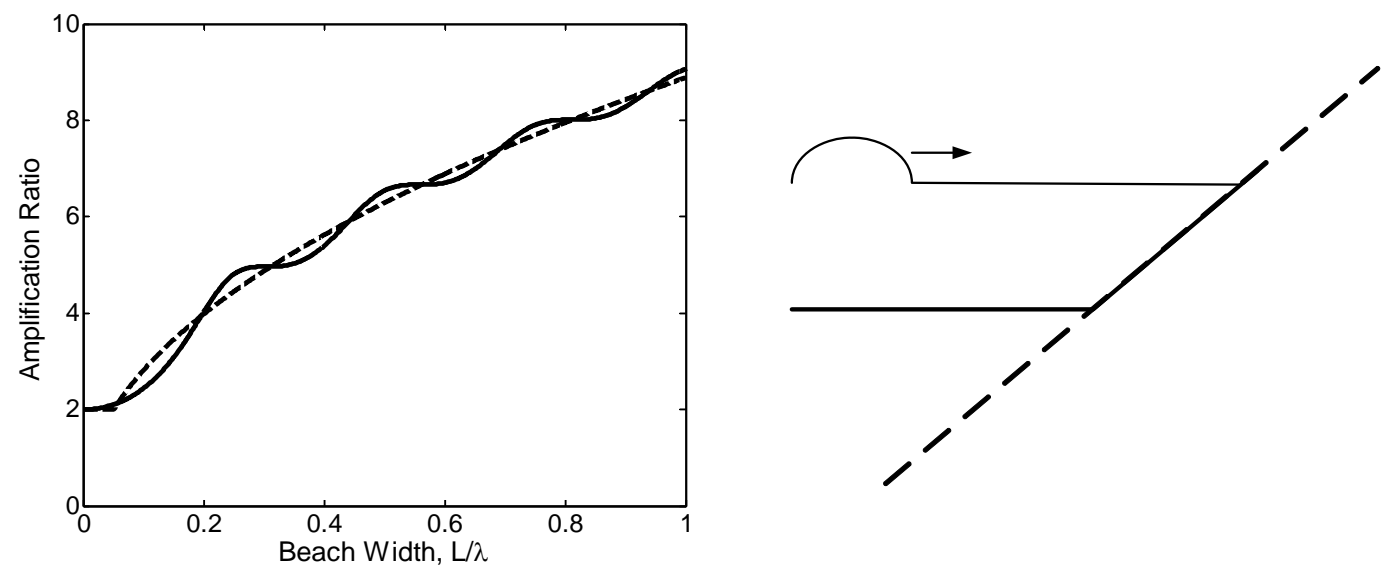

Fig. 1. Amplification ratio for nonlinear shallow water theory above a beach of constant slope (dashed line) and linear shallow water theory for a basin of constant depth, joining a plane beach (solid line).

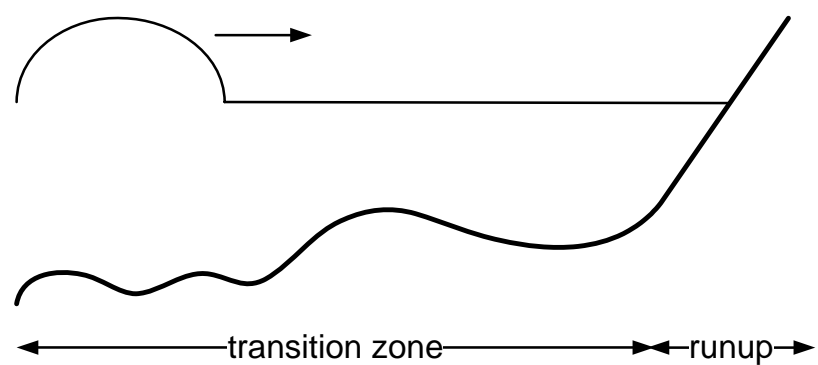

Fig. 2. Sketch of bottom geometry.

simplified geometry of a flat bottom joining a plane beach (a popular geometry of the wave tank in laboratory), this process has been investigated in details (Pelinovsky, 2006; Madsen and Furhman, 2008). In particular, an amplification ratio (a ratio of maximum runup height to initial wave height) versus beach width for a sine wave within such geometry is presented in Fig. 1. The dashed line corresponds to the solution of nonlinear shallow water theory above a beach of constant slope (if the initial wave is linear) and solid line corresponds to the solution of the linear shallow water theory for a basin of constant depth, joining a plane beach. It follows from Fig. 1 that the difference between these solutions, which is determined by reflection from the joining point, is not significant and it allows us to conduct the analysis of the offshore wave transformation process independently of the analysis of wave runup on a coast.

This important conclusion has been used by Didenkulova et al. (2007), who considered independently the nonlinear wave evolution and runup in a basin of constant depth joining a plane beach. Parameters of nonlinear deformed waves above a flat bottom are used as input in the Carrier-Greenspan solution of the runup problem. It has been shown that a nonlinear deformed asymmetric wave penetrates inland over larger distances and with greater velocities than a symmetric one.

The same approach is applied in this study for a more realistic bottom profile, when a slowly varying bottom joins a plane beach near shore (Fig. 2). The paper is organized as follows. Wave propagation and transformation in a basin of slowly varying depth is studied in Sect. 2. An approximate solution of the nonlinear shallow water theory, which allows one describing wave characteristics, is obtained. An increase in wave steepness during the nonlinear wave propagation in a basin of a slowly varying bottom using initial wave characteristics and parameters of the bottom profile is studied in Sect. 3 where detailed analysis is carried out for four different types of the bottom profile: beach of constant depth, a plane beach, a convex and concave beach profiles. It is shown that a wave propagating along a concave beach profile becomes steeper and breaks sooner than for other profiles. The runup problem of these transformed waves on a plane beach is studied in Sect. 4. All results are summarized in the conclusion.

\section{Nonlinear wave transformation in a basin of slowly varying depth}

Basic equations describing nonlinear long wave dynamics in shallow water are

$\frac{\partial \eta}{\partial t}+\frac{\partial}{\partial x}[(h+\eta) u]=0 \quad \frac{\partial u}{\partial t}+u \frac{\partial u}{\partial x}+g \frac{\partial \eta}{\partial x}=0$,

where $\eta(x, t)$ is water displacement, $u(x, t)$ is depthaveraged velocity, $h(x)$ is water depth, $g$ is gravity acceleration, $x$ is a coordinate, and $t$ is time. It is convenient to introduce the Riemann invariants (Stoker, 1957)

$I_{ \pm}=u \pm 2(\sqrt{g(h+\eta)}-\sqrt{g h})$. 
From Eq. (2) it follows that

$u=\frac{I_{+}+I_{-}}{2}, \quad \sqrt{g(h+\eta)}=\frac{I_{+}-I_{-}}{4}+\sqrt{g h}$.

Using Eqs. (2) and (3), the system (1) can be re-written as

$\frac{\partial I_{ \pm}}{\partial t}+\left(\frac{3 I_{ \pm}}{4}+\frac{I_{\mp}}{4} \pm \sqrt{g h}\right) \frac{\partial I_{ \pm}}{\partial x}=\mp\left(\frac{3 I_{ \pm}}{4}+\frac{I_{\mp}}{4}\right) \sqrt{\frac{g}{h}} \frac{d h}{d x}$.

Note that the system (4) is exact for basins of arbitrary variable depth.

Let us consider the transformation of a nonlinear wave (for example, "right-going" wave, which corresponds to the function $I_{+}$), in a basin of a slowly varying depth, when $d h / d x \sim \alpha \ll 1$. In this case the water depth $h$ should be considered as a function of $X=\alpha x$ and Eqs. (4) can be re-written in the following form

$$
\begin{aligned}
& \frac{\partial I_{+}}{\partial t}+\left(\frac{3 I_{+}}{4}+\frac{I_{-}}{4}+\sqrt{g h}\right) \frac{\partial I_{+}}{\partial x}=-\alpha\left(\frac{3 I_{+}}{4}+\frac{I_{-}}{4}\right) \sqrt{\frac{g}{h}} \frac{d h}{d X}, \\
& \frac{\partial I_{-}}{\partial t}+\left(\frac{3 I_{-}}{4}+\frac{I_{+}}{4}-\sqrt{g h}\right) \frac{\partial I_{-}}{\partial x}=\alpha\left(\frac{3 I_{-}}{4}+\frac{I_{+}}{4}\right) \sqrt{\frac{g}{h}} \frac{d h}{d X} .
\end{aligned}
$$

The "left-going" wave $\left(I_{-}\right)$is generated due to reflection in a basin with a smooth bathymetry and it is proportional to the parameter $\alpha \ll 1: I_{-}=\alpha R$, where $R(x, t)$ in the lowest order of the perturbation theory satisfies

$\frac{\partial R}{\partial t}+\left(\frac{I_{+}}{4}-\sqrt{g h}\right) \frac{\partial R}{\partial x}=\frac{I_{+}}{4} \sqrt{\frac{g}{h}} \frac{d h}{d X}$.

Taking into account the smallness of the reflected wave $I_{-}$ Eq. (5) transforms to

$\frac{\partial I_{+}}{\partial t}+\left(\sqrt{g h}+\frac{3 I_{+}}{4}+\frac{\alpha R}{4}\right) \frac{\partial I_{+}}{\partial x}=-\alpha \frac{3 I_{+}}{4} \sqrt{\frac{g}{h}} \frac{d h}{d X}$,

with the same accuracy on the parameter $\alpha$.

Equation (8) still contains the reflected wave and is not independent from Eq. (7). The next approximation is the weakness of the wave field: $\eta=\varepsilon \eta^{\prime}, u=\varepsilon u^{\prime}$, where $\varepsilon \ll 1$ characterizes the ratio of wave height to the water depth. Thus, $I_{+}=\varepsilon I_{+}^{\prime}$. From Eq. (7) it follows that $R=\varepsilon R^{\prime}$. Here we analyze the case when small parameters $\varepsilon$ and $\alpha$ are of the same order $\varepsilon \sim \alpha$ and use only one of them. In this case Eq. (8) transforms to (primes are omitted)

$\frac{\partial I_{+}}{\partial t}+\left(\sqrt{g h}+\frac{3 \varepsilon I_{+}}{4}\right) \frac{\partial I_{+}}{\partial x}=-\varepsilon \frac{3 I_{+}}{4} \sqrt{\frac{g}{h}} \frac{d h}{d X}$,

with an accuracy of the first order of the perturbation theory. Equation (9) contains only one function $I_{+}$and describes the transformed wave only. In the lowest order of the perturbation method this function $I_{+}$can be expressed through the wave flow or water displacement

$u=I_{+} / 2, \quad \varepsilon u=2(\sqrt{g(h+\varepsilon \eta)}-\sqrt{g h})$, and Eq. (9) can be re-written for the velocity

$\frac{\partial u}{\partial t}+\left(\sqrt{g h}+\frac{3 \varepsilon u}{2}\right) \frac{\partial u}{\partial x}+\frac{3 \varepsilon u}{4} \sqrt{\frac{g}{h}} \frac{d h}{d X}=0$.

After introducing new variables

$t^{\prime}=t-\tau(x), \quad X=\varepsilon x, \quad \tau(x)=\int \frac{d x}{\sqrt{g h}}$,

and a new velocity function

$U(t, X)=u(t, X) \frac{h^{3 / 4}(X)}{h_{0}^{3 / 4}}$,

where $h_{0}$ is a depth at the point $x=0$, Eq. (11) transforms into (primes are omitted)

$\left(\frac{h}{h_{0}}\right)^{7 / 4} \frac{\partial U}{\partial X}+\frac{3}{2} \frac{U}{g h_{0}} \frac{\partial U}{\partial t}=0$.

If we again change the variable

$y=\int\left(\frac{h_{0}}{h}\right)^{7 / 4} d X$,

Eq. (14) can be re-written in its final form

$\frac{\partial U}{\partial y}+V(U) \frac{\partial U}{\partial t}=0, \quad V(U)=\frac{3}{2} \frac{U}{g h_{0}}$.

The solution of Eq. (16) satisfying the initial condition $U(t, y=0)=U_{0}(t)$ is

$U(t, y)=U_{0}[t-V(U) y]$.

The implicit Eq. (17) describes a simple or Riemann wave, which is well known in nonlinear acoustics (Rudenko and Soluyan, 1977; Engelbrecht et al., 1988; Gurbatov et al., 1991). The same solution with different modifications has been obtained for water waves (Burger, 1967; Varley et al., 1971; Gurtin, 1975; Pelinovsky, 1982; Caputo and Stepanyants, 2003). Equation (17) allows the description of the deformation of weakly nonlinear wave "velocity" or wave of water displacement (with the use of Eq. 10). The wave shape varies with distance and its steepness increases due to the difference in speed of the crest and trough.

The time derivative of $U(t, y)$ can be calculated in the explicit form

$\frac{\partial U}{\partial t}=\frac{U_{0}^{\prime}}{1+y V_{0}^{\prime}}$,

where the prime means derivative with respect to $t-V(U) y$, and $V_{0}(t)$ is determined through the initial function $U_{0}(t)$ using Eq. (16).

In the case of a wave of elevation approaching the coast at the face slope of the incident wave $\partial u / \partial t<0$, the time derivative of $\partial V_{0} / \partial t<0$ is also negative, and the denominator in Eq. (18) decreases with distance. As a result, the 


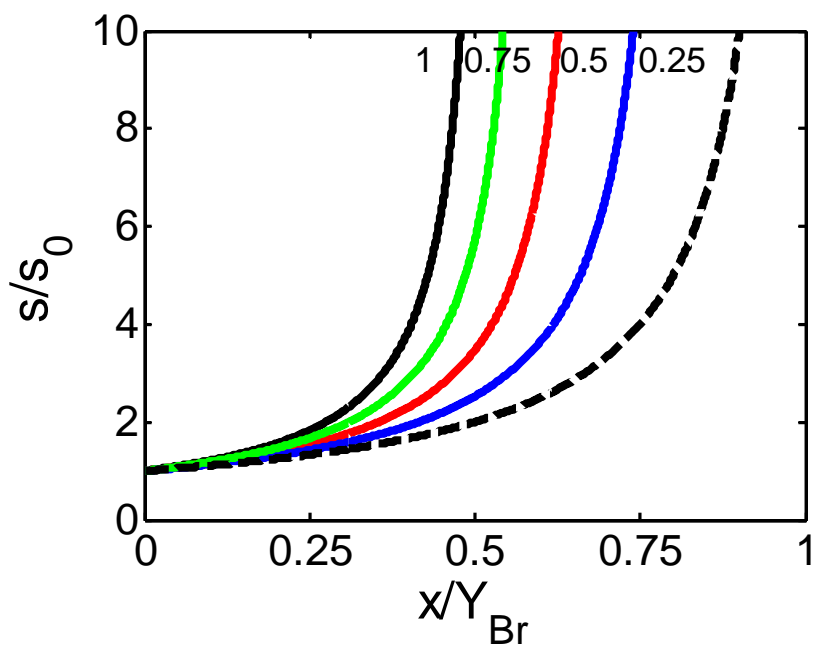

Fig. 3. Wave steepness plotted against distance, in a basin of constant slope, for different values of $Q_{1}$ (solid lines) and a basin of constant depth (dashed line).

time derivative $\partial U / \partial t$ increases and tends to infinity with distance, that allows us finding the distance of the first wave breaking

$Y_{B r}=\frac{1}{\max \left(-d V_{0} / d t\right)}=\frac{2 g h_{0}}{3 \omega a}$.

In the case of a wave of depression the distance of the first wave breaking is described by the same Eq. (19). The difference is in the location of the steep slope of the wave shape only.

As an example, here we analyze the nonlinear deformation of the initial sine wave with "velocity" amplitude $a$ and frequency $\omega$. Due to a weak nonlinearity, using Eq. (10), Eq. (17) can be converted into the expression for water surface displacement

$\eta(t, x)=\sqrt{\frac{h(x)}{g}} u(t, x)$,

and the final expression for the water displacement can be expressed as

$\eta(t, x)=\left(\frac{h_{0}}{h(x)}\right)^{1 / 4} \eta_{0}\left(t-\tau(x)+\frac{3 \eta y(x)}{2 h_{0} \sqrt{g h_{0}}}\left(\frac{h(x)}{h_{0}}\right)^{1 / 4}\right)$,

$y(x)=\int\left(\frac{h_{0}}{h(x)}\right)^{7 / 4} d x$,

where $\eta_{0}$ is an initial shape of water displacement.

Using Eqs. (21) the "face-slope" steepness of the wave can be calculated explicitly

$s=\max (\partial \eta / \partial x)=\left(\frac{h_{0}}{h}\right)^{3 / 4} \frac{s_{0}}{1-y / Y_{B r}}$.
Equations (17), (21) and (22) can be used for computing wave characteristics in the process of wave propagation as it approaches the coast.

\section{Examples of wave deformation for various bottom profiles}

Let us consider several examples of the wave transformation over a distance $L$ in a basin of variable depth, varied from an initial depth $h_{0}$ to a final depth $h_{1}\left(h_{1}<h_{0}\right)$

$h(x)=h_{0}-\left(h_{0}-h_{1}\right)\left(\frac{x}{L}\right)^{b}$.

Here the shape of bottom profile is characterized by power $b$.

In the general case, the integral in Eq. (21) can be expressed through the Hypergeometric function ${ }_{2} F_{1}$ (Gradshtein and Ryzhik, 1965)

$y(x)=$

$$
\frac{x\left[(3 b-4)\left(1-\frac{h_{0}-h_{1}}{h_{0}}\left(\frac{x}{L}\right)^{b}\right)^{3 / 4}{ }_{2} F_{1}\left(\frac{1}{b}, \frac{3}{4}, 1+\frac{1}{b}, \frac{h_{0}-h_{1}}{h_{0}}\left(\frac{x}{L}\right)^{b}\right)+4\right]}{3 b\left(1-\frac{h_{0}-h_{1}}{h_{0}}\left(\frac{x}{L}\right)^{b}\right)^{3 / 4}}
$$

+ const

Here only some special cases, when Eq. (24) can be expressed through primitive functions, are analyzed.

For a basin of constant slope $b=1$

$y(x)=\frac{4 Y_{B r}}{3 Q_{1}\left(1-Q_{1} x / Y_{B r}\right)^{3 / 4}}-\frac{4 Y_{B r}}{3 Q_{1}}$,

$s(x)=\frac{s_{0}}{\left(1+4 / 3 Q_{1}\right)\left(1-Q_{1} x / Y_{B r}\right)^{3 / 4}-4 / 3 Q_{1}}$,

$Q_{1}=\frac{h_{0}-h_{1}}{h_{0}} \frac{Y_{B r}}{L}=\beta \frac{Y_{B r}}{h_{0}}$,

where $\beta$ is the bottom slope and $Y_{B r}$ is the distance of the first wave breaking in a basin of constant depth $h_{0}$, see Eq. (19). Figure 3 illustrates increase of the wave steepness with distance, for different bottom slopes (different values of parameter $Q_{1}$ ).

The steepness of the wave increases with $Q_{1}$, which is proportional to the bottom slope $\beta$, and the wave above a steep bottom profile breaks earlier. The breaking distance $X_{B r}^{1}$ for a beach of constant slope can be found from Eq. (25)

$\frac{X_{B r}^{1}}{Y_{B r}}=\frac{1}{Q_{1}}\left(1-\left(1+\frac{3}{4} Q_{1}\right)^{-4 / 3}\right)$,

(this function is displayed on Fig. 4). For $Q_{1}=0(\beta=0)$, it coincides with the distance of the first breaking in a basin of constant depth, and decreases with an increase in bottom slope. 


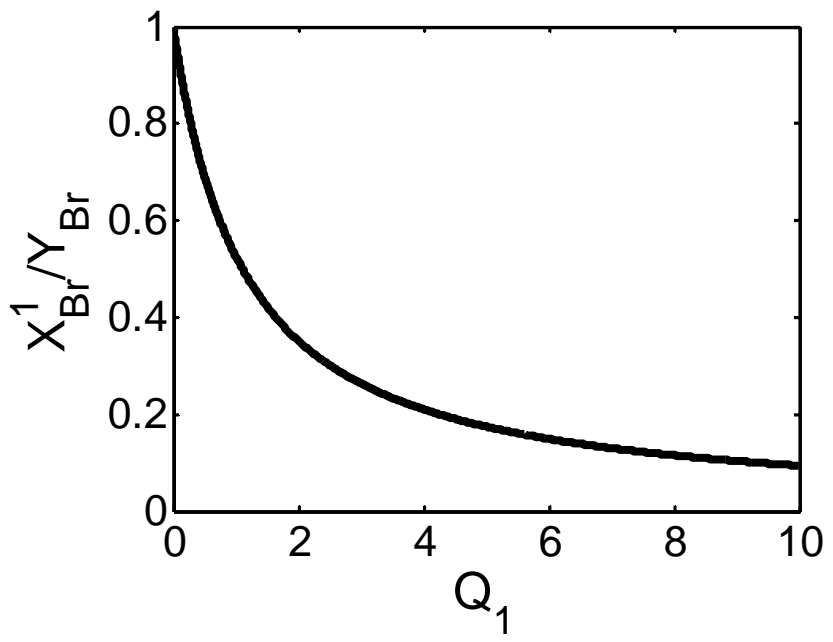

Fig. 4. Breaking distance for the beach of constant slope.

For a convex beach profile, $b=4 / 3$ design formulas are

$y(x)=\frac{x}{\left(1-Q_{4 / 3}\left(\frac{x}{Y_{B r}}\right)^{4 / 3}\right)^{3 / 4}}$,

$$
\begin{aligned}
& s=\frac{s_{0}}{\left(1-Q_{4 / 3}\left(\frac{x}{Y_{B r}}\right)^{4 / 3}\right)^{3 / 4}-\frac{x}{Y_{B r}}}, \\
& Q_{4 / 3}=\frac{h_{0}-h_{1}}{h_{0}}\left(\frac{Y_{B r}}{L}\right)^{4 / 3} .
\end{aligned}
$$

After introducing parameter $Q_{4 / 3}$ the function $s(x)$ has a similar behavior to the function in Fig. 3. Analogously, from Eq. (28) we can find a breaking distance $X_{B r}^{4 / 3}$ for a convex beach profile as a function of parameter $Q_{4 / 3}$

$\frac{X_{B r}^{4 / 3}}{Y_{B r}}=\frac{1}{\left(1+Q_{4 / 3}\right)^{3 / 4}}$

and the derived function looks similar to Fig. 4. The wave steepness increases with an increase in parameter $Q_{4 / 3}$ and the wave breaks earlier than for a beach of constant depth.

In the case of a concave beach shape with $b=1 / 2$ design formulas are

$y(x)=\frac{8 Y_{B r}\left(4-3 Q_{1 / 2} \sqrt{\frac{x}{Y_{B r}}}\right)}{3 Q_{1 / 2}^{2}\left(1-Q_{1 / 2} \sqrt{\frac{x}{Y_{B r}}}\right)^{3 / 4}}-\frac{32 Y_{B r}}{3 Q_{1 / 2}^{2}}$,

$s=\frac{s_{0}}{\left(1+\frac{32}{3 Q_{1 / 2}^{2}}\right)\left(1-Q_{1 / 2} \sqrt{\frac{x}{Y_{B r}}}\right)^{3 / 4}-\frac{8}{3 Q_{1 / 2}^{2}}\left(4-3 Q_{1 / 2} \sqrt{\frac{x}{Y_{B r}}}\right)}$,

$Q_{1 / 2}=\frac{h_{0}-h_{1}}{h_{0}} \sqrt{\frac{Y_{B r}}{L}}$.

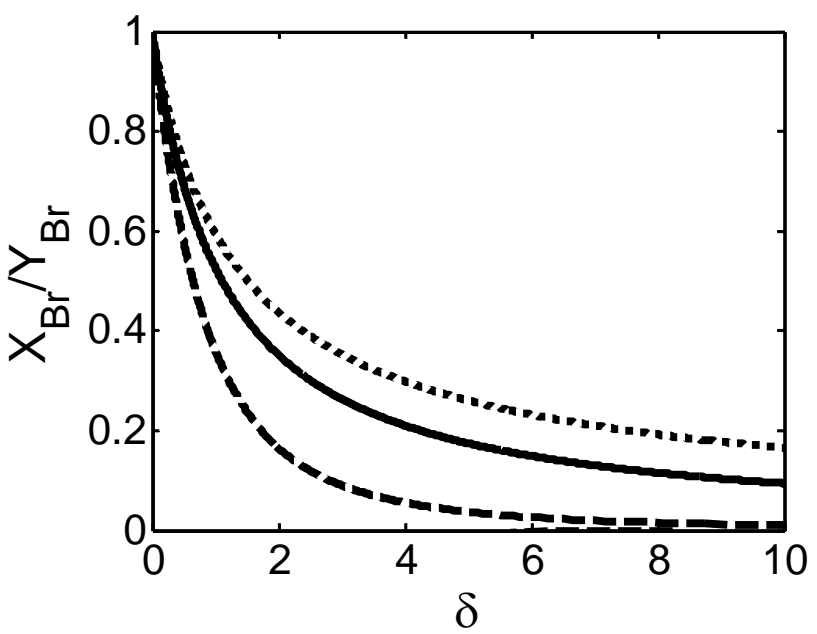

Fig. 5. Breaking distance plotted against depth variation $\delta$; plane beach (solid line), convex (dotted) and concave (dashed) bottom profiles; $Y_{B r} / L=1$.

The behavior of function $s(x)$ is qualitatively the same as that shown in Fig. 3. The breaking distance $X_{B r}^{1 / 2}$ for a concave bottom profile as a function of parameter $Q_{1 / 2}$ cannot be presented in an explicit form

$\left(3 Q_{1 / 2}^{2}+32\right)\left(1-Q_{1 / 2} \sqrt{\frac{X_{B r}^{1 / 2}}{Y_{B r}}}\right)^{3 / 4}=8\left(4-3 Q_{1 / 2} \sqrt{\frac{X_{B r}^{1 / 2}}{Y_{B r}}}\right)$,

but nevertheless the behavior of this function is similar to analogous functions for a plane beach and a convex bottom profile.

An influence of the bottom shape on the breaking distance is demonstrated on Fig. 5 for a fixed ratio $Y_{B r} / L=1$. A new parameter $\delta=\left(h_{1}-h_{0}\right) / h_{0}$ characterizes the depth variations with distance $L$. An increase in the power $b$ of the bottom profile leads to an increase in its breaking distance and it is therefore slower developing nonlinear deformation. Thus the wave breaks sooner, propagating along a concave bottom profile, than for a plane or convex beach. This can be explained in terms of an average depth of the transition zone, which is less for a concave profile.

Variations of the breaking distance for different beach profiles with the ratio $L / Y_{B r}$ are shown on Fig. 6 for a depth change of $10 \%(\delta=0.1)$. The figure also demonstrates that the wave breaks sooner in a basin of a concave bottom profile than on a plane or convex beach.

Due to the nonlinearity, the wave can break in the transition zone also (Fig. 2). Mathematically, the condition of the first wave breaking (wave steepness tends to the infinity) corresponds to the zero value of the denominator in Eqs. (26), (29) and (32). It allows one finding the relation between initial wave amplitude $A_{0} / h_{0}$ and the characteristics of the bottom shape in a transition zone of variable depth; see Fig. 7. The wave breaks if its amplitude is big or the length of the 


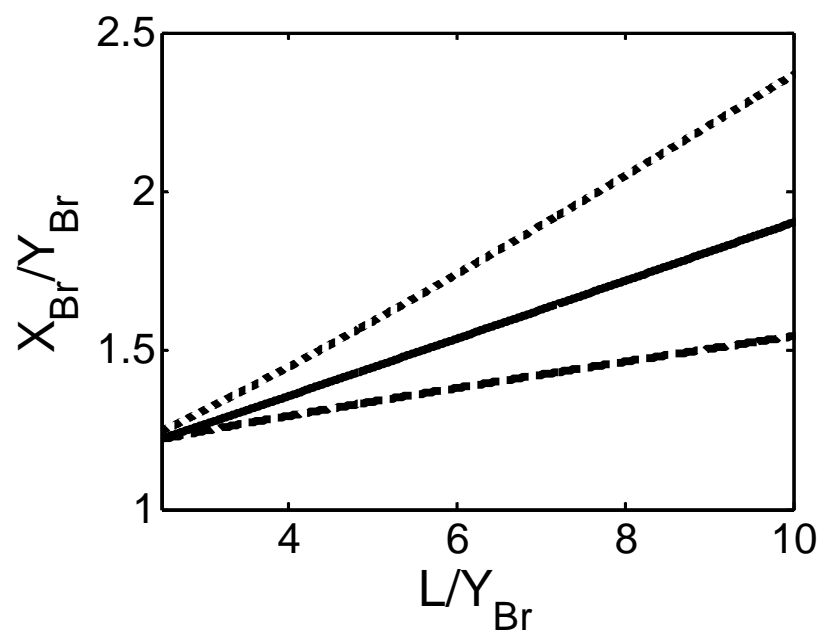

Fig. 6. Breaking distance and the ratio $L / Y_{B r}$ for a plane beach (solid line), and convex (dotted) and concave (dashed) bottom profiles; $\delta=0.1$.

transition zone is large. That is why, with the same conditions, a wave on a concave beach (which has greater amplification) breaks sooner than on a convex beach, what explains the behavior of the curves presented in Fig. 7.

\section{Runup of deformed waves on a coast}

The runup of the nonlinear deformed waves approaching the coast is now considered. We assume that the bottom profile in the vicinity of the shoreline can be approximated by a linear function (beach of constant slope). This condition is required for using the Carrier-Greenspan approach (Carrier and Greenspan, 1958) for the rigorous solving of nonlinear shallow-water equations. We also assume that the beach width is relatively large when considering an approaching wave as a wave of weak (but finite) amplitude. In this case, all formulas for initial conditions of a runup problem can be given in explicit form (in variables $x$ and $t$ instead of the Carrier-Greenspan variables $\sigma$ and $\lambda$ ); see (Didenkulova et al., 2007). The last assumption is that initial conditions in the Carrier-Greenspan transformation give an incident wave in the form of a nonlinear deformed wave as presented in Sect. 3 and neglect wave reflection from the inflection point between transmitted and runup zones. Arguments for this assumption, based on analysis of previous results summarized in Pelinovsky (2006); Madsen and Furhman (2008), are given in the Introduction. These approximations are used in Didenkulova et al. (2007) considering the long wave runup on a plane beach joining a flat bottom. The principal result obtained in this study shows a strong influence of the incident wave steepness on the runup characteristics (runup height, maximum velocity, conditions of the wave breaking).
Here we consider a plane beach joint with a transition zone of variable depth (Fig. 2). Characteristics of the approaching wave are calculated in Sect. 3. Taking into account the fact that the maximum runup characteristics can be found from linear theory (Synolakis, 1991; Didenkulova et al., 2007), the runup of periodic waves of an arbitrary shape can be described by the following Fourier series (Didenkulova et al., 2007)

$$
\begin{aligned}
R_{\text {shore }}(t)= & 2 \pi \sqrt{\frac{2 D}{\lambda}} H_{0} R e \sum_{n=1} \sqrt{n} B_{n} \exp \left[i\left(n \omega t+\frac{\pi}{4}\right)\right], \\
U_{\text {shore }}(t)= & (2 \pi)^{2} H_{0} \sqrt{2 \frac{g}{h_{1}}\left(\frac{D}{\lambda}\right)^{3}} \operatorname{Re} \sum_{n=1} n^{3 / 2} \\
& B_{n} \exp \left[i\left(n \omega t+\frac{3 \pi}{4}\right)\right] .
\end{aligned}
$$

Here $R_{\text {shore }}(t)$ and $U_{\text {shore }}(t)$ are vertical oscillations of the shoreline and its horizontal speed computed within the linear theory (extremes of both functions coincides with maximum values of runup characteristics in the nonlinear theory (Synolakis, 1991; Didenkulova et al., 2007)),

$\lambda=2 \pi \sqrt{g h_{1}} / \omega$

is a wavelength of an incident wave at the distance $D$ from the shoreline with a water depth $h_{1}$ (the point where plane beach of constant slope, $\alpha$ joins a transition zone), $H_{0}$ and $B_{n}$ are an amplitude of the first harmonic and dimensionless spectral amplitudes of an incident wave respectively given at the same point

$\eta(t)=H_{0} R e \sum_{n=1} B_{n} \exp (i n \omega t)$,

where Re means the real part of the complex sum. In general, Eqs. (34) and (35) should include a time shift which is the travel time to the shoreline, but this is ignored, since it does not influence the maximum runup characteristics.

The wave breaking on a beach of constant slope occurs if parameter $B r=1$, where

$$
\begin{aligned}
B r= & (2 \pi)^{3} \frac{H_{0}}{h_{1}} \sqrt{2\left(\frac{D}{\lambda}\right)^{5}} \max \operatorname{Re} \sum_{n=1} n^{5 / 2} \\
& B_{n} \exp \left[i\left(n \omega t+\frac{5 \pi}{4}\right)\right] .
\end{aligned}
$$

These formulas are valid for periodic waves of an arbitrary shape. If an incident wave is the Riemann wave, the maximum runup height can be expressed as a function of the wave steepness (Didenkulova et al., 2007)

$R_{\max }=2 \pi H_{0} \sqrt{\frac{2 D}{\lambda} \frac{s}{s_{0}}}$. 


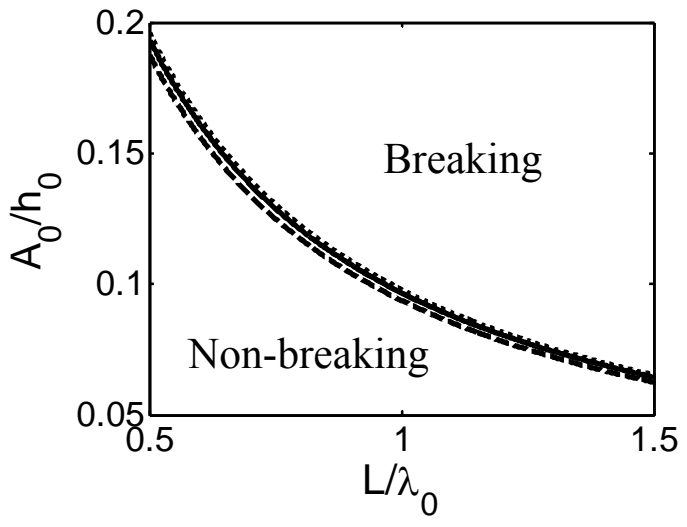

(a) $h_{1} / h_{0}=0.9$

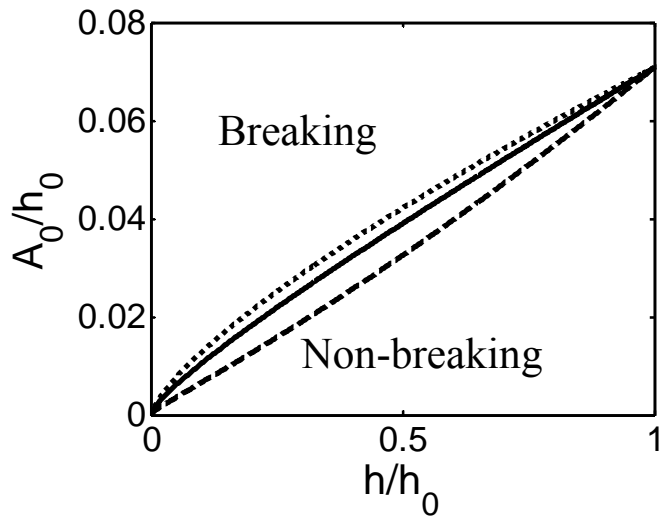

(b) $L / \lambda_{0}=1.5$

Fig. 7. Breaking and non-breaking regimes of the wave transformation in the transition zone; plane beach (solid line), convex (dotted) and concave (dashed) bottom profiles.

The main advantage of Eq. (39) is its universal form. It contains only wave characteristics (amplitude, wavelength and steepness) at the beginning of the runup stage, and does not depend on the wave evolution in the transition zone if the ratio between spectral components is the same as in local Riemann solution. That is why we can apply Eq. (39) for the analysis of the runup of the quasi-Riemann wave described in Sect. 3.

In the case of a plane beach, with the use of Eq. (26), Eq. (39) transforms to

$$
R_{\max }^{1}=2 \pi A_{0}\left(\frac{h_{0}}{h_{1}}\right)^{1 / 2} \sqrt{\frac{2 D}{\lambda_{0}\left[1-\frac{4 \pi L}{\lambda_{0}} \frac{A_{0}}{h_{0}} \frac{\left(h_{0} / h_{1}\right)^{3 / 4}-1}{1-h_{1} / h_{0}}\right]}},
$$

in the case of a convex bottom, and with the use of Eq. (29), it transforms to

$$
R_{\max }^{4 / 3}=2 \pi A_{0}\left(\frac{h_{0}}{h_{1}}\right)^{1 / 2} \sqrt{\frac{2 D}{\lambda_{0}\left[1-\frac{3 \pi L}{\lambda_{0}} \frac{A_{0}}{h_{0}}\left(\frac{h_{0}}{h_{1}}\right)^{3 / 4}\right]}} .
$$

For a concave bottom, with the use of Eq. (32), it changes to

$$
\begin{aligned}
R_{\max }^{1 / 2}= & 2 \pi A_{0}\left(\frac{h_{0}}{h_{1}}\right)^{1 / 2} \\
& \sqrt{\frac{2 D}{\lambda_{0}\left[1-\frac{8 \pi L}{\lambda_{0}} \frac{A_{0}}{h_{0}} \frac{\left(1+3 h_{1} / h_{0}\right)\left(h_{0} / h_{1}\right)^{3 / 4}-4}{\left(1-h_{1} / h_{0}\right)^{2}}\right]}} .
\end{aligned}
$$

It follows from Eqs. (40-42), the amplification ratio (maximum runup height to initial wave amplitude) depends on four parameters: dimensionless initial wave amplitude $A_{0} / h_{0}$, the depth ratio $h_{1} / h_{0}$, the width $L / \lambda_{0}$ of the transition zone and the width of the runup zone $D / \lambda_{0}$. It should be pointed that $\lambda_{0}$ in Eqs. (40-42) is the length of an incident wave in the open ocean.

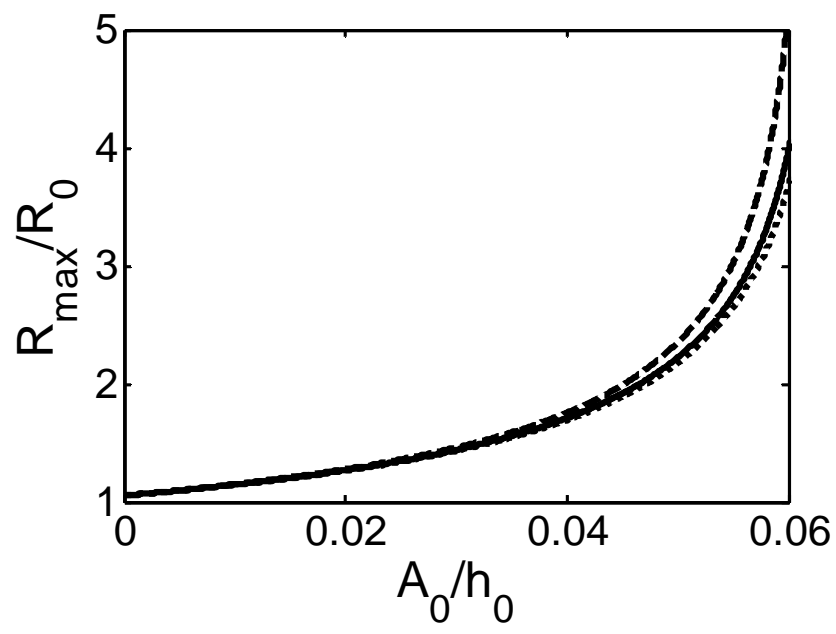

Fig. 8. Maximum runup height $R_{\max } / R_{0}$ plotted against the amplitude of an initial wave $A_{0} / h_{0}$; plane beach (solid line), convex (dotted) and concave (dashed) bottom profiles.

In real conditions if we consider a water basin with initial depth $h_{0}=1 \mathrm{~km}$ changing to $h_{1}=100 \mathrm{~m}$ over distance, $L=400 \mathrm{~km}$ and then with a plane beach to the coast over the distance $D=10 \mathrm{~km}$, the maximum runup height of the wave with amplitude $A_{0}=10 \mathrm{~cm}$ and wavelength $\lambda_{0}=5 \mathrm{~km}$ is $R_{\max }^{1}=5.71 \mathrm{~m}$ for a transformation zone of constant slope, $R_{\max }^{4 / 3}=5.24 \mathrm{~m}$ for a convex and $R_{\max }^{1 / 2}=9.41 \mathrm{~m}$ for a concave transformation zone.

The dependence of the maximum runup height $R_{\max } / R_{0}$ on amplitude $A_{0} / h_{0}$ for a fixed depth ratio $h_{1} / h_{0}=0.9$ and a width of the transition zone $L / \lambda_{0}=1.5$ is presented in Fig. 8. Here $R_{0}=2 \pi A_{0} \sqrt{2 D / \lambda_{0}}$ corresponds to the maximum runup of a sine wave with amplitude $A_{0}$ on a sloping beach. The wave runup on a plane beach joining a concave profile is higher than for a plane or a convex beach. This can 


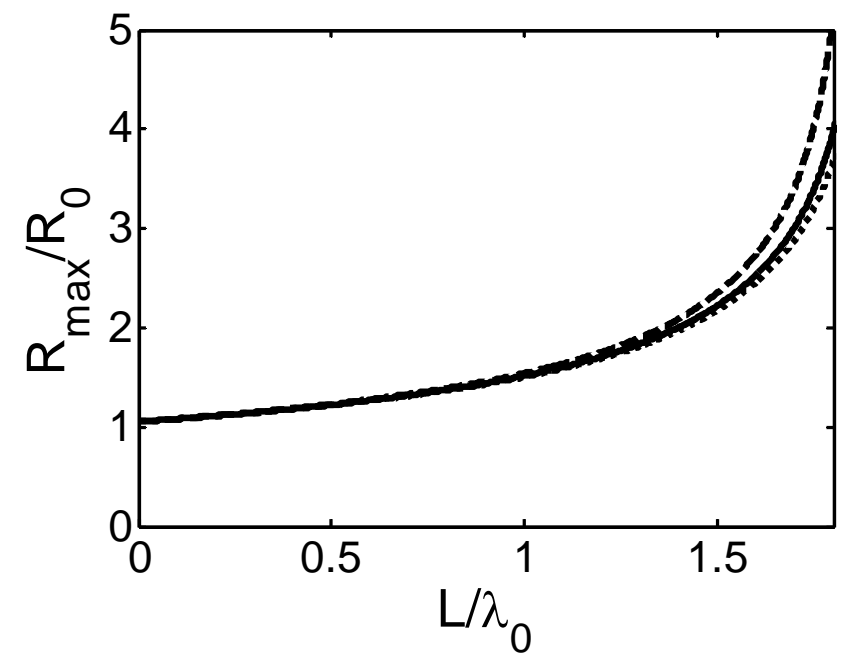

Fig. 9. Maximum runup height $R_{\max } / R_{0}$ plotted against the width of transmission zone $L / \lambda_{0}$; plane beach (solid line), convex (dotted) and concave (dashed) bottom profiles, $h_{1} / h_{0}=0.9, A_{0} / h_{0}=0.05$.

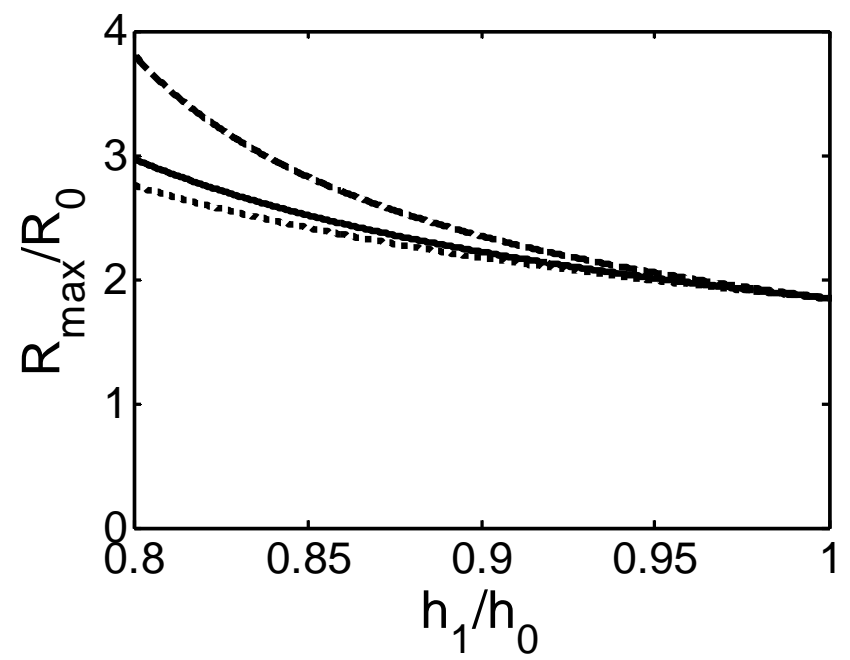

Fig. 10. Maximum runup height $R_{\max } / R_{0}$ plotted against the depth ratio $h_{1} / h_{0}$; plane beach (solid line), convex (dotted) and concave (dashed) bottom profiles, $A_{0} / h_{0}=0.05, L / \lambda_{0}=1.5$.

be explained by larger "average" nonlinearity and a smaller equivalent depth, which lead to greater wave steepness on a concave beach.

The same effect can be seen in Figs. 9 and 10, which illustrate dependences of the runup height on the width of the transition zone and on the depth ratio respectively. It appears that wave steepness is the most significant parameter characterizing the runup process.

The above theory is valid only for non-breaking waves. The breaking parameter determined by Eq. (38) is also a function of the dimensionless initial wave amplitude $A_{0} / h_{0}$, the depth ratio $h_{1} / h_{0}$, the width of the transition zone $L / \lambda_{0}$

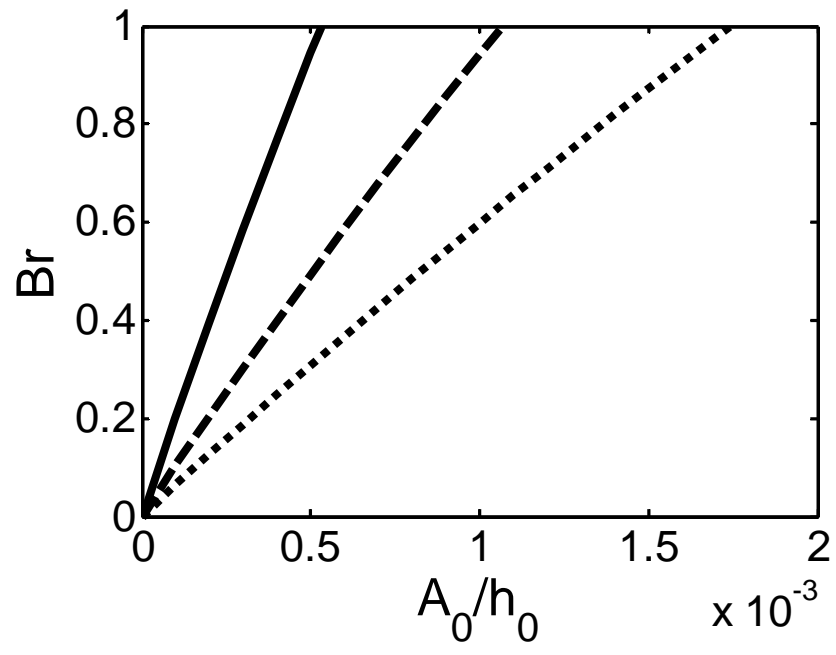

Fig. 11. Breaking parameter versus initial wave amplitude for $L / \lambda_{0}=2, D / \lambda_{0}=1.1$, solid, dashed and dotted lines correspond to $h_{1} / h_{0}=0.7,0.8$ and 0.9 , respectively.

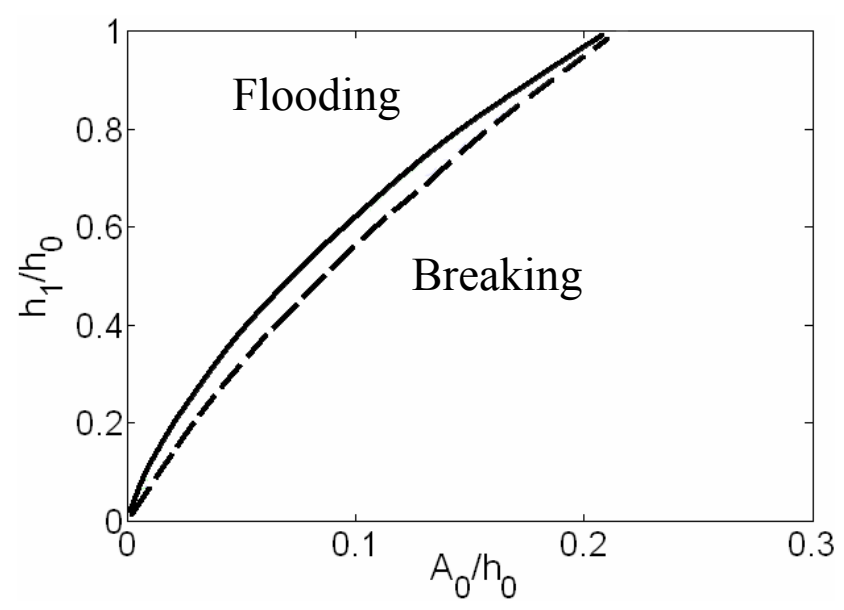

Fig. 12. Various scenarios of the wave runup on a coast (dashed line corresponds to a plane beach and solid line to a concave beach).

and the width of the runup zone $D / \lambda_{0}$. The dependence of the wave breaking parameter $B r$ on initial wave amplitude and the depth ratio is displayed in Fig. 11 for $L / \lambda_{0}=2$ and $D / \lambda_{0}=1.1$. A significant difference in depth along a transition zone leads to an increase in parameter $\mathrm{Br}$.

The condition $B r=1$ separates two important scenarios of the wave runup: surging or flooding (without breaking) for $B r<1$, and plunging (breaking) for $B r>1$. The separation of these regimes on a plane $\left(h_{1} / h_{0}, A_{0} / h_{0}\right)$ for $L / \lambda_{0}=1.5$ and $D / \lambda_{0}=1.1$ is displayed in Fig. 12. A concave beach is more "nonlinear" (the averaged depth is smaller), and that is why the wave breaking on it occurs for smaller amplitudes and greater depth ratio $\left(h_{1} / h_{0}, A_{0} / h_{0}\right)$, than for a plane beach. 


\section{Conclusion}

The problem of long wave shoaling and runup on the coast is described in the framework of 1-D nonlinear shallow-water theory. The bottom geometry considered in the paper consists of the transition zone with a slowly varying depth (in the scale of the wavelength) and a beach of constant slope in the vicinity of the shoreline. An incident wave in the open ocean has a sinusoidal shape and small amplitude. In the transition zone, the wave shoaling is described by an asymptotic solution in the form of the quasi-Riemann wave with varying amplitude. The wave steepness and spectral components are calculated for three bottom profiles: a beach of constant slope, a convex and a concave profiles. It is shown that a concave profile is more "nonlinear" as its average depth along the wave path is smaller than for other profiles. The wave runup on a plane beach is studied in the framework of the Carrier-Greenspan approach with a nonlinear deformed wave in the transition zone as an input wave. The runup characteristics and the condition of wave breaking are analyzed with respect to the wave parameters in the open ocean. It is demonstrated that wave steepness is the most significant parameter characterizing the runup process. The comparison between different transition zones shows that in the general case, the concave beach gives a larger increase in the wave steepness and greater amplification of the wave amplitude. At the same time waves propagating along such a beach break sooner.

\section{Appendix A}

\section{Spectral presentation of the Riemann wave}

In practice it is important to know the Fourier frequency spectrum of the wave field. The spectral presentation of the Riemann wave can be presented explicitly (Pelinovsky, 1982; Didenkulova et al., 2006b):

$$
\begin{aligned}
& U(t, y)=\sum_{n=1}^{\infty} U_{n}(y) \sin (n \omega t), \\
& U_{n}(y)=2 a \frac{Y_{B r}}{n y} J_{n}\left(\frac{n y}{Y_{B r}}\right)=\frac{4 g h_{0}}{3 \omega y n} J_{n}\left(\frac{3 n \omega y a}{2 g h_{0}}\right),
\end{aligned}
$$

where $J_{n}$ is the Bessel function of $n$-order. After substituting our original variables from Eq. (13) we have the final expression for the velocity field

$$
\begin{aligned}
& u(t, x)=\left(\frac{h_{0}}{h(x)}\right)^{3 / 4} \sum_{n=1}^{\infty} U_{n}(y) \sin (n \omega t), \\
& y(x)=\int\left(\frac{h_{0}}{h(x)}\right)^{7 / 4} d x .
\end{aligned}
$$

Due to a weak nonlinearity, using Eq. (20), Eq. (A2) can be converted into the expression for water surface displacement

$$
\begin{aligned}
\eta(t, x) & =\sum_{n=1}^{\infty} A_{n}(x) \sin (n \omega t) \\
& =2 A_{0}\left(\frac{h_{0}}{h(x)}\right)^{1 / 4} \sum_{n=1}^{\infty} \frac{Y_{B r}}{n y} J_{n}\left(\frac{n y}{Y_{B r}}\right) \sin (n \omega t),
\end{aligned}
$$

where $A_{0}=a \sqrt{h_{0} / g}$ is an initial amplitude of water displacement. Note that $t$ in Eqs. (A1-A3) is a time in a reference system of coordinates $t-\tau(x)$. Nevertheless, a travel time $\tau$ does not influence the energetic characteristics of the wave process and it is omitted.

If $h(x)=h_{0}$ Eqs. (A2) and (A3) reduce to the known equations for wave transformation in the basin of constant depth (Didenkulova et al., 2006b, 2008).

Using Eq. (22) for the "face-slope" steepness of the wave spectral amplitudes in Eq. (A3) can be re-written as functions of the face slope steepness

$$
A_{n}=\frac{2 A_{0}\left(\frac{h_{0}}{h}\right)^{1 / 4}}{n\left(1-\frac{s_{0}}{s}\left(\frac{h_{0}}{h}\right)^{3 / 4}\right)} J_{n}\left[n\left(1-\frac{s_{0}}{s}\left(\frac{h_{0}}{h}\right)^{3 / 4}\right)\right] \text {. }
$$

If we introduce an effective amplitude $A_{\text {eff }}$ and steepness $s_{\text {eff }}$

$$
A_{\text {eff }}=A_{0}\left(\frac{h_{0}}{h}\right)^{1 / 4}, \quad s_{\mathrm{eff}}=s\left(\frac{h}{h_{0}}\right)^{3 / 4}=\frac{s_{0}}{1-y / Y_{B r}},
$$

spectral amplitudes of the nonlinear deformed wave in a basin of slowly varying depth can be presented in a universal form, which coincides with an expression for a basin of constant depth (Didenkulova et al., 2007)

$A_{n}=\frac{2 A_{\mathrm{eff}}}{n\left(1-\frac{s_{0}}{s_{\mathrm{eff}}}\right)} J_{n}\left[n\left(1-\frac{s_{0}}{s_{\mathrm{eff}}}\right)\right]$.

Acknowledgements. This research is supported by Marie Curie network SEAMOCS (MRTN-CT-2005-019374), EEA grant (EMP41) and RFBR (08-05-00069, 08-05-91850, 08-05-72011, 08-02-00039). The author would like to thank Kevin Parnell for his help in preparing the final version of the manuscript. Author thanks the editor Efim Pelinovsky, Narcisse Zahibo and the anonymous reviewer for their useful comments and suggestions.

Edited by: E. Pelinovsky

Reviewed by: N. Zahibo and another anonymous referee

\section{References}

Bugge, T., Belderson, R., and Kenyon, N.: The Storegga slide, Phil. Trans. P. Soc. Lond. A, 325, 357-398, 1988.

Burger, W.: A note on the breaking of waves on non-uniformly sloping beach, J. Mech. and Math., 16(10), 1131-1142, 1967. 
Caputo, J.-G. and Stepanyants, Y. A.: Bore formation, evolution and disintegration into solitons in shallow inhomogeneous channels, Nonlin. Processes Geophys., 10, 407-424, 2003, http://www.nonlin-processes-geophys.net/10/407/2003/.

Carrier, G. F. and Greenspan, H. P.: Water waves of finite amplitude on a sloping beach, J. Fluid Mech., 4, 97-109, 1958.

Choi, B. H., Pelinovsky, E., Kim, K. O., and Lee, J. S.: Simulation of the trans-oceanic tsunami propagation due to the 1883 Krakatau volcanic eruption, Nat. Hazards Earth Syst. Sci., 3, 321-332, 2003,

http://www.nat-hazards-earth-syst-sci.net/3/321/2003/.

Didenkulova, I. I. and Pelinovsky, E. N.: Run up of long waves on a beach: the influence of the incident wave form, Oceanology, 48(1), 1-6, 2008a.

Didenkulova, I. and Pelinovsky, E.: Run up of long waves on a beach: the influence of the incident wave form, Pure Appl. Geophys., 165(11-12), 1-6, doi:10.1007/s00024-008-0425-6, 2008b.

Didenkulova, I. I., Slunyaev, A. V., Pelinovsky, E. N., and Kharif, C.: Freak waves in 2005, Nat. Hazards Earth Syst. Sci., 6, 10071015, 2006a, http://www.nat-hazards-earth-syst-sci.net/6/1007/2006/.

Didenkulova, I. I., Zahibo, N., Kurkin, A. A., Levin, B. V., Pelinovsky, E. N., and Soomere, T.: Runup of nonlinearly deformed waves on a coast, Doklady Earth Sciences, 411(8), 1241-1243, 2006b.

Didenkulova I., Pelinovsky, E., Soomere, T., and Zahibo, N.: Runup of nonlinear asymmetric waves on a plane beach, Tsunami and Nonlinear Waves, edited by: Kundu, A., Springer, 173-188, 2007.

Didenkulova, I. I., Pelinovsky, E. N., and Soomere, T.: Exact travelling wave solutions in strongly inhomogeneous media, Estonian J. Eng., 14(3), 220-231, 2008.

Glimsdal, S., Pedersen, G. K., Atakan, K., Harbitz, C. B., Langtangen, H. P., and Lovholt, F.: Propagation of the Dec. 262004 Indian Ocean Tsunami: effects of dispersion and source characteristics, Int. J. Fluid Mech, Res., 33(1), 14-33, 2006.

Gradshtein, I. S. and Ryzhik, I. M.: Tables of Integrals, Sums, Series, and Products, Academic Press, New York, 1965.

Gurtin, M. E.: On the breaking of water waves on a sloping beach of arbitrary shape, Quart. Appl. Math., 33, 187-189, 1975.

Hills, J. G. and Gods, M. P.: Tsunami from asteroid and comet impacts: the vulnerability of Europe, Sci. Tsunami Hazards, 16, 3-10, 1998.

Kharif, Ch., Pelinovsky, E., and Slunyaev, A.: Rogue waves in the ocean, Springer, 2009.

Kim, K. O., Lee, H. S., Yamashita, T., and Choi, B. H.: Wave and storm surge simulations for Hurricane Katrina using coupled process based models, KSCE Journal of Civil Engineering, 12(1), 1-8, 2008.

Le Mehaute, B., Koh, R. C., and Hwang, L. S.: A synthesis of wave run-up, J. Waterways Port Harbor Coastal Eng., 94, 77-92, 1968.

Le Mehaute, B.: Engineering methods: run-up, surge on dry-bed, energy dissipation of tsunami waves, in: Tsunamis, edited by: Hwang, L. S. and Lee, Y. K., 156-181, 1979.
Lay, Th., Kanamori, H., Ammon, Ch. J., Nettles, M., Ward, S. N., Aster, R. C., Beck, S. L., Bilek, S. L., Brudzinski, M. R., Butler, Rh., DeShon, H. R., Ekström, G., Satake, K., and Sipkin, S.: The Great Sumatra-Andaman Earthquake of 26 December 2004, Science, 308, 1127-1133, 2005.

Mader, C. L.: Modeling the La Palma Island tsunami, Sci. Tsunami Hazards, 19(3), 150-170, 2001.

Madsen, P. A. and Fuhrman, D. R.: Run-up of tsunamis and long waves in terms of surf-similarity, Coastal Engineering, 55, 209223, 2008.

Pararas-Carayannis, G.: Evaluation of the threat of mega tsunami generation from postulated massive slope failures of island strato-volcanoes on La Palma, Canary Islands, and on the island of Hawaii, Sci. Tsunami Hazards, 20(5), 251-277, 2002.

Parnell, K., Delpeche, N., Didenkulova, I., Dolphin, T., Erm, A., Kask, A., Kelpsaite, L., Kurennoy, D., Quak, E., Räämet, A., Soomere, T., Terentjeva, A., Torsvik, T., and Zaitseva-Pärnaste, I.: Far-field vessel wakes in Tallinn Bay, Estonian J. Eng., 14(4), 273-302, 2008.

Pelinovsky, E. N.: Nonlinear Dynamics of Tsunami Waves, Applied Physics Institute Press, Gorky, 1982.

Pelinovsky, E.: Hydrodynamics of tsunami waves. Chapter 1, in: Waves in Geophysical Fluids, edited by: Grue, J. and Trulsen, K., CISM Courses and Lectures, No. 489, Springer, 1-48, 2006.

Pelinovsky, E., Choi, B., Stromkov, A., Didenkulova, I., and Kim, H.: Analysis of tide-gauge records of the 1883 Krakatau tsunami, Tsunamis: case studies and recent developments, Adv. Nat. Technol. Hazards Res., Springer, 57-77, 2005.

Simkin, T. and Fiske, R. S.: Krakatau 1883 - the volcanic eruption and its effects, Smithsonian Institution Press, Washington, D.C., 1983.

Soomere, T.: Nonlinear components of ship wake waves, Appl. Mechanics Rev., 60, 120-138, 2007.

Stockdon, H. F., Holman, R. A., Howd, P. A., and Sallenger, A. H.: Empirical parameterization of setup, swash, and runup, Coast. Engineering, 53, 573-588, 2006.

Stoker, J. J.: Water waves, Willey Inter Science, NY, 1957.

Synolakis, C. E.: The runup of solitary waves, J. Fluid Mech., 185, 523-545, 1987.

Synolakis, C. E.: Tsunami runup on steep slopes: How good linear theory really is, Nat. Hazards, 4, 221-234, 1991.

Varley, E., Venkataraman, R., and Cumberbatch, E.: The propagation of large amplitude tsunamis across a basin of changing depth. I. Off-shore behaviour, J. Fluid Mech., 49(4), 775-801, 1971.

Ward, S. and Asphaug, E.: Asteroid impact tsunami: a probabilistic hazard assessment, Icarus, 145, 64-78, 2000.

Ward, S. N. and Day, S.: Cumbre Vieja volcano - Potential collapse and tsunami at La Palma, Canary Island, Geophys. Res. Lett., 28(17), 3397-3400, 2001.

Zahibo, N., Didenkulova, I., Kurkin, A., and Pelinovsky E.: Steepness and spectrum of nonlinear deformed shallow water wave, Ocean Engineering, 35(1), 47-52, 2008. 